Unlike freckles, however, chloasma may depend upon the existence of serious internal disease. It may, as in one of the cases before us, follow an infectious malady, as measles. It may occur as a result of malarial intoxication. It sometimes co-exists with malaria, tuberculosis or cancer. Chloasma may develop in consequence of mental excitement. Certain affections of the skin also give rise to chloasma. Among these I may mention senile atrophy, morphea and scleroderma. Popular opinion has long associated chloasma with hepatic derangement, and a common name for the affection is liver spot. The most usual internal cause of the anomalous pigmentation is disorder of the uterus or ovaries. When due to this cause, the affection is known as chloasma uterinum. This form locates itself particularly upon the forehead and temples. In some instances the discoloration is very deep. It is apt to be especially dark in brunettes. Sometimes there are a number of small brown patches. In other cases the pigmentation extends almost without a break from ear to ear. Chloasma of utero-ovarian origin, forms an exception to the rule that only the face is involved. A deepening of the normal color may be seen around the nipples. In some cases the discoloration is not of long duration. In others it continues for an indefinite period. The surface is smooth, unless complicated with some other cutaneous malady. In pregnancy we sometimes meet with cases in which the discolored patches are very large and, indeed, involve almost the entire surface.

Chloasma uterinum may occur either in the single or married, either as a result of disease or of pregnancy. It does not of ten occur after the grand climacteric has been reached. Not infrequently it is associated with hysteria. When the underlying cause has been cured the chloasma generally disappears.

It is not possible to mistake freckles for any other affection. Any one is perfectly competent to make the diagnosis. There is one disease, however, which bears some resemblance to chloasma, and that is tinea versicolor. The patches of the latter malady usually appear upon the body, those of chloasma upon the face. In tinea there are a number of spots; in chloasma, there are but few. Tinea versicolor is accompanied by a fine scaling. The surface is perfectly smooth in chloasma and there is no desquamation. The patches of tinea enlarge rapidly and itch. Chloasma spreads slowly and gives rise to no itching. Finally, the microscope furnishes us with a test. Tinea versicolor is caused by the growth of a specific vegetable organism, the presence of which can be readily recognized.

Treatment. - The treatment of chloasma depends upon the same principles as that of freckles. The list of drugs which we may employ locally is much the same in both affections. In chloasma, however, we may be obliged to take measures for the improvement of the general health or for the relief of visceral disorders. Chloasma uterinum demands a therapy addressed to the genital system. Again, we must be cautious in the use of external remedies, remembering that certain caustics, when used too freely, invite a deposit of pigment. This is especially true of mustard, Spanish fly and croton oil. The mineral acids are also open to the same objection. Carbolic acid may be mopped lightly upon the surface of the discolored patch with advantage. Acetic acid may be likewise used in the same way. The local application of the tincture of iodin will sometimes prove beneficial. Caustic potash, potash and soda soaps may also be of avail. Veratrin ointment, the ointment of the nitrate of mercury or of ammoniated mercury, or mercurial ointment are other preparations which have their advocates. I have generally derived more satisfaction, however, from the employment of the ointment of copper oleate, the mercuric or the mercurous oleate. The corrosive sublimate was a favorite with Hebra, who used it dissolved in distilled water, alcohol or collodion, in the proportion of five grains to the ounce. A compress moistened in the solution is kept applied to the affected surface for a period of four hours. This produces a blister and the discolored epiderm is replaced by one free from pigment, though the color may subsequently reappear. Brushing the patch with peroxid of hydrogen will sometimes answer a good purpose. The tincture of benzoin and borax are also of service in chloasma. These various agents may be combined as in the manner indicated for freckles. A prescription which I have often used is thus composed:

R. Hydrarg. chlorid corros. . Ammon. chlorid. . . Sp. vini rectificat. m.$$
\text { Aq. hamamelid dest. . . f f }
$$

Better than all the medicinal applications named, in both freckles and chloasma, is the use of the galvanic current. I am constantly in the habit of making use of this treatment and can testify, from an extended experience, to its advantage. Galvanism stimulates absorption of the pigment and is, for that reason, far preferable to the use of drugs, after which the pigment is often reproduced in the new cuticle. The results from galvanism are more uniformly permanent.

THE IMPORTANCE OF EARLY RECOGNITION AND TREATMENT OF OBSTRUCTIVE DISEASES OF THE UPPER RESPIRATORY

$$
\text { TRACT. }
$$

Read before the Philadelphia County Medical Society, March 14, 1894. BY JOSEPH S. GIBB, M.D.

NSTRUCTOR IN JARYNGOLOGY IN THE PHILADELPHIA POLYCLINIC; SURGEON-IN-CHARGE OF THE EAR, NOSE, AND THROAT DEPARTMENT OF THE EPISCOPAL HOSPITAL, PHILADELPHIA.

The introduction of a subject so time-worn and stale might require an apology were it not that notwithstanding there is a plethora of literature there still remains a singular indifference and lack of appreciation of the merits of the matter in the mind of the general practitioner. Doubtless much of this is due to the very voluminous character of the literature, which tends rather to interest those for whom the subject has an especial attraction. We read much of the histologic structure of the various hypertrophies, and numerous are the instruments introduced for their destruction. We read less of the best means of demonstrating their existence, the etiologic factors concerned in their production, and the prophylactic measures which may be adopted to prevent their development.

Perhaps no single abnormal condition is productive of more marked alterations in physical beauty and healthy functions than diseases producing obstruction in the upper respiratory tract. The anxious 
and watchful mother views with sorrow the freckling or tanning of her child's face. How much more disagreeable than these are alterations in the facial $w$ lines to such degree as to cause a face to be expressionless : and when to this is added partial deafness, with all its unpleasant effects, we have a picture which any careful mother should strive to avoid. Were as much care bestowed on the important functions of the nasal chambers as is generally given to the skin or other tissues more directly under the eye, we would have fewer of these unpleasant pictures. Nor is this marring of physical beauty the sole deleterious result of these conditioris; it can be proven that serious and perhaps grave interference with important functions follow in its wake.

In order to have a proper appreciation of the importance of the subject we will review for a moment, and in a cursory manner, the anatomic structure and physiologic purposes of the nasal chambers and their contiguous structures.

In the bony nasal chambers are three scroll-like bodies, the turbinate bones, two of which, the middle and inferior, are accessible in the living subject, which form the sides of the chamber; a septum, separating the nasal chambers, which is reinforced by a plate of cartilage in the recent state. These turbinated bones divide the nasal chambers into three meatuses-superior, middle, and inferior-into which open the various sinuses or foramina which communicate with the accessory cavities-namely, the frontal sinus, the ethmoid and sphenoid cells, the antrum of Highmore, and also the lachrymal duct. All these structures in the living subject are covered with mucous membrane well endowed with blood vessels and nerves, whilst over the turbinate bones the tissue is erectile or cavernous.

The situation of these chambers, the peculiar construction of the bones, the unusually large surface of mucous membrane for so small a cavity, and the very generous blood supply, bespeak important functions. Bosworth claims three; 1 the first and very important function in respiration; 2 , an aid in phonation by acting as a resonant chamber; 3 , as an olfactory organ.

The atmospheric air as it passes through the nasal chambers is brought in contact with a comparatively large surface of mucous membrane which is rendered warm by the free blood supply and moist from the secretion of the numerous mucous glands therein contained. The effect of this contact is first to ab. stract from the air, dust and other foreign bodies, and secondly to add to the inspired air warmth and. moisture. This latter function is most important. It has been computed that over 7,000 grains of water are expired from the lungs in twenty-four hours; and Aschenbrandt has demonstrated by a series of carefully conducted experiments that the source of this moisture is in the nasal chambers.

From this brief and very imperfect glance at the anatomical position and functional purposes of the nasal chambers we may draw a few important lessons. Interference with the proper and easy inspiration of air through the nasal chambers is productive of diseased conditions, not alone of those organs themselves but of the more important respiratory structures within the chest.

It can not be doubted that inspiration of air into the air vesicles imperfectly warmed, deprived of the moisture which Nature intended it should have, must exercise a deleterious influence on the delicate mucous membrane lining these cells. I am not ready with statistics to prove the relationship between pul. monary and bronchial affection and obstructive diseases of the upper air tract, nor do I deem it necessary, for it is a matter of common experience and observation that those children whose nasal chambers are obstructed either directly by hypertrophies, polyps, etc., or indirectly by hypertrophied pharyngeal or faucial tonsils, are sunken-chested, prone to attacks of acute bronchitis and coryza, and in many respects are less healthy than other children. I am well aware that these conditions are often the result of a constitutional vice which in itself renders the subject less robust, still I am more than ever convinced that removal of the local effects of this diathetic condition results more effectually in a return to a normal condition than any plan of treatment directed solely to the condition itself. Nor is it alone the respiratory tract that suffers from these abnormal states. I have before remarked that children so afflicted are apt to be in a condition of poor health generally, and it has occurred to me that perhaps this may be not entirely due to the diathetic condition which is so frequent a concomitant, but rather that the lunge, being supplied constantly with a vitiated air, $i . e$, air insufficiently warmed and moistened, failed to supply the blood with a healthy, proper pabulum and hence the tissues suffer. By the explanation what it may, we must not lose sight of the fact that a condition of ill health frequently does exist, and we should bend our energies to ascertain the cause and seek its remedy.

It is not the object of this paper to elaborate on the various methods of treatment, but rather to inquire in to the causation and to endeavor to point ont, means which may be taken to ameliorate the conditions existing and to prevent the development of more serious ones. Especially is it desired to inquire into the causative influences of obstructive diseases of the upper air passages in young children.

Eliminating all those effects of imperfect or perverse fetal development such as occlusion of anterior or posterior nares, cleft palate, etc., we have conspicuously prominent in the category of obstructive lesions in the upper respiratory tract-hypertrophic conditions of the turbinate bodies, of the pharyngeal, faucial, and to a lesser degree the lingual tonsil.

A glance at the records of any of our large throat and nose clinics will convince one that these abnormal conditions are far from rare; nor does even this showing give us a thoroughly accurate conception of their frequency. Too common is it to ignore or carelessly treat the various catarrhal symptoms presented to $u s$, and this indifference $" \|$ the part of the profession has led to a corresponding indifference on the part of the laity, so that only those cases of pronounced obstruction are brought to the notice of the general practitioner. Were the nasal speculum used as freely as the stethoscope or clinical thermometer we would be astonished at the result. Again, certain hypertrophic conditions have but comparatively recently received any measure of attention from the profession. Though faucial hypertrophies have been observed and studied from remote times, it was not until a very recent date that Meyer gave to the profession a clear idea of the significance of hypertrophic conditions of that collection of adenoid tissue 
at the vault of the pharynx known as the pharyngeal tonsil; nor is the importance of the subject to-day appreciated by the rank and file of the profession, notwithstanding that much has been written about it and many instruments devised for its removal. Situated as it is in a somewhat inaccessible or rather unobservable portion of the upper pharynx, it is only by the use of the rhinoscopic mirror that its presence can be clearly demonstrated; this being the case, it is patent that it must be frequently overlooked.

With these facts before us it is clear that, whereas our clinics show us that these states are common, they give us no adequate conception of their prevalence.

At the clinic of the Episcopal Hospital it is the custom to carefully examine the nasal cavity, the pharynx, and post-nasal surfaces in every case that applies for treatment, irrespective of the fact that the subjective symptoms point to lesions in this or that locality; and in children of tender age, where a rhinoscopic examination is not feasible, if there is any suggestion of disease in this locality a finger is introduced back of the sof palate and over the vault of the pharynx to demonstrate by tactile sense the presence or absence of any abnormality. It is surprising as a resuit of this procedure to note the number of cases in which there are lesions of the upper pharynx in which the symptoms have been overlooked by the parents.

In considering the etiology of these hypertrophies it is as well to treat of them collectively, for whereas they are often separate and distinct lesions and exist independently, they are often associated and their etiology, so far as our knowledge goes, is in many respects similar.

In the case of the glandular tissue there can be no doubt that in a fer instances these hypertrophies are congenital, doubtless the effects of an inherited vice-syphilis or scrofula-carrying out the wellknown tendency of these dyscrasiæ to affect lymphatic and glandular tissue. Though this view is combated by no less an authority than Meyer,who leans to the view that it is always an inflammatory process in a tissue which normally is largely developed in infancy and childhood, at the same time it is difficult to explain the presence of this state in very young infants unless we presume this inflammatory process to have taken place during its pre-natal existence. Those cases developing subsequent to birth doubtless have as a starting-point an inflammatory or catarrhal origin.

We have dimonstrated the frequency of hypertrophies of the glandular tissues and turbinates in children, and have admitted a few cases may be congenital, which leaves the vast majority to have its development subsequent to birth; and, as it is our belief that its causation lies in some condition outside of the child, at least in part, it is now our endeavor to seek out those causes and, if possible, lay down certain laws looking to their eradication or, at least, amelioration. Here, as with many other diseases of insidious approach, an early recognition is productive of much good.

Among the general causative agencies which it is not entirely within our power to relieve, may be mentioned atmospheric conditions incident to residence in temperate climes, and especially near the seaboard; the method of heating houses in cities by dry air frequently derived from an unsanitary source; carelessness in the dressing of infants and trusting them too much to the care of servants who unnecessarily expose them. All these and others exercise an iufluence in the production of the disease, but only in a general way. The prophylactic remedies are obvious and suggest themselves.

The various catarrhal conditions which the above enumerated disturbing influences of our environment occasion, are of much importance from an etiologic standpoint. There can be no doubt that it. is here we have the starting.point of the hypertrophic process. A simple coryza is neglected; the child is not even protected from the causes which produced it-the result, one coryza succeeding another the entire season. The effect of a long-continued inflammatory process is precisely the same in the nasal and pharyngeal tissues as in the other tissues of the body -namely, increase in the connective-tissue growth, increase in the cellular growth. And it is just here by a knowledge of the effects that the family physician's advice and council can be of so much value and save so much misery and suffering to the childso much mental anxiety and mortification to the parents. I believe that parents should be taught that a coryza is by no means so simple or harmless an affair as is commonly believed. It is better to err on the right side-better by far to give some attention (possibly needless) to a few attacks of coryza than to sit quietly by doing nothing, and permitting the production of obstructive masses in the upper airpassages that may alter the child's expression and possibly injure the health.

Parents should further be taught that repeated coryzas indicate a diseased condition of the nasal or pharyngeal mucous membrane demanding prompt and thorough treatment. The physician should himself insist on making a careful examination aided by the nasal speculum and rhinoscopic mirror, and should lay down a plan of treatment which his ingenuity will suggest, and persevere with it until the conditions yield; and I believe in this way, and in this way alone can we hope to have any measure of success.

Conspicuous in the category of special causative agencies may be mentioned the eruptive diseases of childhood, and especially those in which the fauces and nasal chambers are involved in the diseased process. Foremost among these may be mentioned diphtheria. The well-known tendency of diphtheritic processes to attack glandular tissue is well exemplified in the post-nasal spaces. We are familiar with the appearance of the diphtheritic membrane on the tonsils, but usually the examination of the other mucous surfaces is not made, and we remain in ignorance as to the extent of this process into the nasal chambers and vault of pharynx. Doubtless if careful examination was carried out in every case we would find the proportion in which these structures are involved to be very large. I have no doubt that many of the cases of so-called re-infection are in reality those in which there has been an involvement of the pharyngeal tonsil in the diphtheritic process. When an examination of the tonsils reveals an absence of the familiar membrance and a subsidence of the inflammatory condition, we are lulled into a sense of security and perhaps relax in our vigilance, unmindful of the fact that the disease is perhaps making insidious inroads at a spot beyond our unaided view. However, we are not concerned with this at 
present; sufficient is it for us to know the extreme susceptibility of the mucous surfaces in this disease.

After the subsidence of the diphtheritic process if is very common to have a catarrhal condition continuing for a considerable period; this is especially noticeable in cases in which the nasal mucous membrane has been implicated; though it frequently occurs where there has been no perceptible involvement of the nasal chambers, and I believe it is just in these cases that there has been a diseased process going on at the vault of the pharynx. The explanation of this mucus discharge and other evidences of a catarrhal condition is doubtless due to alterations in the nutrition of the part, in consequence of the high grade of inflammatory action, and also to septic absorption. Were an examination made at this time we would likely find a hypertrophic condition either in the nasal turbinates or the pharyngeal and faucial tonsil. Though doubtless the majority of these cases do well without any special treatment directed to the part, yet in a certain number the catarrhal condition persists, and especially is this likely to occur in those children who seem prone to catarrhal states, and the foundation is laid for the formation of masses of hypertrophied tissue.

The beneficent offices of the family physician can be here admirably employed. It is not wise to pass over lightly the persistence of a catarrhal state after the subsidence of the inflammatory symptoms. Treatment employed at this time and persisted in until an amelioration takes place, can be productive only of good.

Scarlatina, as is well-known, expends at least a part of its energy on the mucous membrane of the fauces; though, unlike diphtheria, it rarely involves the nasal chambers. Here, as in the foregoing, the inflammatory action is so high, often to the production of false membrane, as to exercise a baneful influence upon the nutrition of the tissue and aid in the production of hypertrophies. We are all familiar with the fact that hypertrophies of the faucial tonsils are a frequent sequela of this disease. The same process that gives rise to hypertrophies in this tissue is at the same time exerting its influence on the tissues of the vault.

It is needless to $\mathrm{dw}$ ell on this; the too familiar sight of catarrhal conditions as sequelæ of scarlatina precludes any argument to the contrary as to its position as a causative agent in the conditions under consideration. Nor is it hardly necessary to refer to the necessity for prompt treatment-one word, however, on this point. It seems to me an imperative duty on the part of the medical attendant to insist on an examination and, if necessary, subsequent treatment in every case where a catarrhal condition evidenced by a mucous or muco-purulent discharge from the nose, a thick voice, and mouth-breathing persists, after all evidences of the exanthemata have passed away.

Measles, though commonly regarded as a light and insignificant disease, is exceedingly apt to leave some trace of its existence. The explanation of this is evident. Not commonly menacing life, it is, however, very active in its attack, and this activity is exerted on the mucous surfaces in the entire respiratory tract. Mothers should be informed of the fact that with care the course of the disease will be benign, but without it there is extreme probability of a catarrhal affection with all its unpleasant conse- quences being left as a sequela. And here, as with others, active treatment should be insisted upon if the catarrhal symptoms persist after the subsidence of other evidences of the exanthemata.

'The other exanthemata and febrile diseases, such as rötheln, variola, varicella, typhoid fever, etc., exert a certain amount of influence as causative agents, but only in a general way, as any alteration in health will do. The diseases mentioned, viz., diphtheria, scarlatina and measles, exert a special influence because the diseased condition seems to expend itself on the mucous surfaces, producing alterations and perversion of function.

The clinical picture presented by sufferers from obstructive diseases of the upper respiratory tract is absolutely characteristic, and especially is this true as regards the alteration of facial lines and changes in expression. Naturally this obstruction to the entrance of the air by the normal route, viz., through the nose, necessitates its seeking some other mode of ingress to reach the lungs; the only other way is through the mouth, and hence we have the condition of mouth-breathing with all the discomfort and unpleasant effects which such a condition entails. Long-continued mouth-breathing brings into play muscles about this orifice not usually called upon for excessive work, and at the same time nominally puts at rest muscles about the nose whose functions are not called into play; the result is an alteration of facial expression to such a degree in well-marked cases as to give to the child a stupid, almost idiotic appearance. The obstruction in the case of the pharyngeal tonsil may become so excessive as to encroach on the pharyngeal orifice of the Eustachian tube, interfering with the proper supply of air to the tympanic cavity, thus inducing catarrhal or inflammatory conditions of the mucous lining of this tube and also of the tympanic chamber. The result of this is dullness of hearing or even complete deafness.

The normal course of the secretion from the numerous mucous glands in the nasal chambers is obstructed and hence flows back into the naso-pharynx; added to this we have a greatly increased secretion from the adenoid vegetations at the vault. During sleep this mucus collects in the pharynx, and as the child is obliged to breathe through the mouth, we have associated loud snoring with rattling and gurgling of air through the mucus secretion; hence the child is restless and fitful and the annoyance to other members of the household excessive.

We have then as the salient features of a case we are attempting to portray: Mouth-breathing, snoring, rattling of mucus in the fauces, and disturbed sleep; a dull, listless expression of countenance; hardness of hearing, and in many cases impairment of general health, causing the child to be anemic, sunkenchested, prone to catarrhal attacks, and of stunted growth.

Much has been written and various are the opinions as to the treatment of these distressing conditions. We are not concerned in this article whether it is the proper method to remove adenoid growths by forceps, curette, or finger; to diminish hypertrophied turbinates by means of galvamo-cautery, snare or escharotics, but a few words in conclusion as to the management of cases in their incipiency may not be amiss.

Mothers should be urged not to neglect a persistent snuffle in a child, not to regard as trivial the con- 
tinuance of a mucus discharge after diphtheria or the exanthemata. Physicians, on the other hand, should not pass these complaints lightly by ignoring them, or directing in an indifferent manner some simple and likely inefficient remedy.

The management of hypertrophies at this early stage is as simple as the results are happy. After a careful examination the nares and upper pharynx should be cleansed of mucus by an antiseptic and detergent spray, e.g., Dobell's solution, Seiler's tab. let, Listerin in diluted solution, etc., and then applications made to the affected mucous membrane of an astringent or alterant, depending on the condition of the parts. Should we find simply an increased secretion with no appreciable alteration in the turbinates, tonsils, and surrounding tissues, it is likely we will accomplish all that could be desired by directing spraying of the nares thoroughly by one or other of the solutions mentioned twice daily and continued faithfully until the membrane presents a normal appearance and the secretion ceases.

In cases still farther advanced in which we find the turbinates quite red and somewhat swollen; the pharyngeal tonsil secreting more mucus than normal, and rather large; the faucial tonsil red and somewhat hypertrophied, we will find it advantageous to use some application to diminish the inflammatory condition. These applications may be made quite painless by spraying the parts with a 5 per cent. solution of cocain or introducing a cotton tampon saturated in the same solution. In the nares a solution which has gained favor in the various clinics devoted to these diseases is that of iodin. The strength of the solution may be graded by the severity or obstinacy of the case. It is well to begin with a weak solution, increasing the strength as the necessities of the case demand. In some cases which resist this plan of treatment a touch of the fused bead of chromic acid along the turbinates will often accomplish the desired object; this latter plan should be immediately followed by the antiseptic spray to dilute and limit the destructive power of the escharotic. It is merely necessary in these early cases to touch the mucous membrane lightly to produce a superficial eschar; the resulting slough and subsequent contraction will so squeeze the blood vessels as to bring about the desired diminution in the size of the tissues.

The pharyngeal tonsil also in many cases will respond satisfactorily to applications of iodin on a pledget of cotton carried by an applicator so curved as to pass readily behind the soft palate and up to the vault. Glycerole of tannin has been found to act very happily, though perhaps a little more unpleasant in its effects. The application of chromic acid (fused) to a pharyngeal tonsil should not be advised, though undoubtedly as useful as in the nose, unless the operator is steady and familiar with making applications by the aid of the rhinoscopic mirror.

It would be hardly necessary to refer to the treatment of catarrhal and slight hypertrophic conditions of the faucial tonsils the result of the causes named, were it not that here, in spite of the ready accessibility of the part, they are subject to neglect. The same process of cleansing is as necessary as in the other hypertrophies, after which an application of a solution of $60 \mathrm{gr}$. to the drachm of nitrate of silver, glycerole of tannin, or in stubborn cases producing a superficial slough by fused chromic acid, will probably answer every purpose.
In the more advanced cases or in those which fail to respond to the above-outlined plan of treatment and a steady increase of the hypertrophied tissues occurs, there is but one plan which should be carried out at as early a date as possible, namely a destruction of the hypertrophied tissue. The various plans to accomplish this object will not be touched upon, but can be readily learned by consulting any of the modern treatises devoted to the treatment of diseases of the nose and throat.

In recapitulation the object of this paper has been to call attention to the following points:

1. The importance and desirability of early attention to diseases of the upper respiratory tract consisting of the nose and naso-pharynx.

2. The production of alteration in nutrition and possibly pulmonary and bronchial troubles by hypertrophic conditions of the upper respiratory tract.

3 . The etiology of these states referring to the influence of environment and especial mention of diphtheria and the exanthemata as prominent factors in this category.

4. The prevalence of these conditions.

5. Carelessness and indifference in both physician and patient as to the various catarrhal states which are believed to be the precursors of the diseases under consideration.

6. The necessity of early treatment and the power for good exercised by the family physician in these troublesome conditions.

7. The clinical picture presented by these unfortunates, laying especial stress on the alteration in facial expression and the distressing and amnoying condition of the child during sleep.

\section{TREATMENT OF DIPHTHERIA.}

Read before the Wayne County Medical Society, 1894.

$$
\text { BY OSCAR S. ARMSTRONG, M.D. }
$$
DETROIT, MICH.

There is no disease with which we have to contend that requires better surroundings or greater care in its treatment to insure a successful outcome, than diphtheria. It is rightly dreaded as one of the most deadly foes of childhood, and even adults do not escape its attacks. It is a treacherous disease, and one or two members of a family may have it in a mild form, while in the remaining children it may present its most malignant characteristics. Nor does its treacherous character end here, for often when our patient seems to be doing nicely, with membrane entirely gone, pulse and temperature normal and everything progressing favorably, some of its dan. gerous sequelæ make their appearance.

The first record we have of diphtheria in America was the epidemic in Boston in the sixteenth century, since which time it has become completely domiciled. Originally the disease appeared in epidemic form only, but at the present time it has become essentially endemic in nearly all our large centers of population. Its ravages are not confined to the homes of the poor in overcrowded and fil thy tenement houses, but extend to those of the wealthy in country and city. Until the last few years little was understood of its true nature, but pathologists by careful and patient investigation have discovered its cause. Klebs was the first to demonstrate the existence of a specific bacillus of diphtheria, and his conclusions were afterwards confirmed by Löffler. Experiments have proven that 